\title{
Curve Optimization of Tapered Cantilever Beams Under Tip Loads
}

\author{
Minyi Zhu, Guobin Gong* and Jun Xia \\ Department of Civil Engineering, Xi'an Jiaotong-Liverpool University, Suzhou, \\ Jiangsu 215123, China
}

Received 24 June 2020; Accepted (in revised version) 8 December 2020

\begin{abstract}
The study adopts the variational method for analyzing the cantilever tapered beams under a tip load as well as a definite end displacement, and further determining the optimized shapes and materials that can minimize the weights. Two types of beams are taken into account, i.e., the Euler-Bernoulli beam without considering shear deformation and the Timoshenko beam with shear deformation. By using the energy theorem and the reference of isoperimetric problem, the width variation curves and the corresponding minimum masses are derived for both beam types. The optimized curve of beam width for the Euler-Bernoulli beam is found to be a linear function, but nonlinear for the Timoshenko beam. It is also found that the optimized curve in the Timoshenko beam case starts from non-zero at the tip end, but its tendency gradually approaches the one of the Euler-Bernoulli beam. The results indicate that with the increase of the Poisson's ratio, the required minimum mass of the beam will increase no matter how the material changes, suggesting that the optimized mass for the case of Euler-Bernoulli beam is the lower boundary limit which the Timoshenko case cannot go beyond. Furthermore, the ratio $\rho / E$ (density against Elastic Modulus) of the material should be as small as possible, while the ratio $h^{2} / L^{4}$ of the beam should be as large as possible in order to minimize the mass for the case of Euler-Bernoulli beam, of which the conclusion is extended to be applicable for the case of Timoshenko beam. In addition, the optimized curves for Euler-Bernoulli beam types are all found to be power functions of length for both tip point load cases and uniform load cases.
\end{abstract}

AMS subject classifications: 49K35, 49S05, 74P05

Key words: Tapered beam, Euler-Bernoulli beam, Timoshenko beam, variational principle, isoperimetric problem, curve optimization.

${ }^{*}$ Corresponding author.

Emails: Minyi.Zhu19@student.xjtlu.edu.cn (M. Zhu), Guobin.Gong@xjtlu.edu.cn (G. Gong), Jun.Xia@xjtlu. edu.cn (J. Xia) 


\section{Introduction}

Tapered beams, also called non-prismatic beams, are beams with variable cross sections along the longitudinal directions in order to reduce their weights and adapt various stress distribution. Tapered beams are commonly used in many fields, such as airplanes, machinery, bridges and buildings. In a tapered beam, the bending stresses vary along the longitudinal axis and is no longer proportion to the bending moment since the section modulus also varies along the axis [11,14]. Applications of tapered beams under dynamic situations can be found in the literature $[4,13]$. A recent case is found in the literature for the tapered cantilever beam used in piezoelectric energy harvesters, which indicates the significance of tapered shape to provide the maximum efficiency [5].

Recent rapid developments of optimization technology and algorithms allows researchers to optimize tapered beams to a favorable level. Imam [7] explained the basic concept of shape optimization design and described the boundary effects. Dietl and Garcia [2] optimized the beam shapes for harvesting power using a heuristic optimization code, as well as experimental validation. Yoo [18] divided a tapered beam into multiple segments and assumed the width and the thickness as cubic spline functions to optimize. Ohsaki et al. [12], Katsikadelis et al. [8] and Kim et al. [9] optimized Euler-Bernoulli beams considering different load cases including torsional problems with the help of finite element method. Vinot et al. [16] presented a detailed procedure in shape optimization of thin-walled beam-like structures with correction coefficients. However, little literature can be found using variational method and considering shear effects in optimization, which is one of the objectives in this paper

The concept and application of variational methods are widely involved in many fields. The isoperimetric method, as a typical variational problem, has the elementary propositions to find the loop curve with a constant length covering the maximum area, or further determine the shape of a suspension cable. Such problems are normally turned into unconditional extremum problems using the Lagrange multiplier method and then solved using Euler-Lagrange Equation (transferring a variational problem to a calculus problem), see $[1,3,6,10]$.

This study attempts to apply a variational method to optimize the tapering curve of a cantilever tapered beam under a tip load. Two different beam types are taken into considerations, i.e., the Euler-Bernoulli beam without shear deformation and the Timoshenko beam with shear deformation considered. The investigation will focus on the cantilever beam only tapered for the width of its cross section while keeping the same height of its cross section along the longitudinal axis, but the overall cross section is still kept as a rectangular shape (see Fig. 1). The results of this investigation could be utilized to optimize the tapering curve in order to minimize the mass with fixed tip displacements, e.g., for light-weight tapered beam designs in bridges and airplanes. 


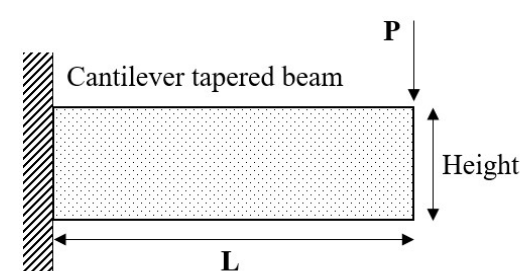

(a). Side view

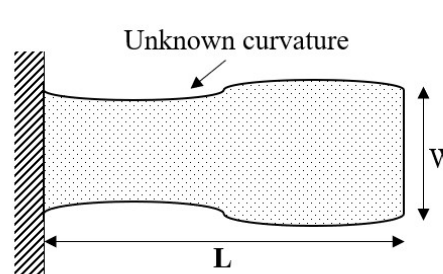

(b). Plan view

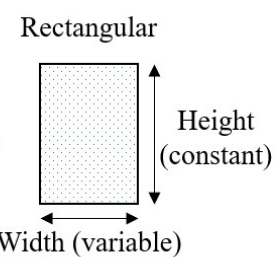

(c). Cross-section

Figure 1: Cantilever beam only tapered for the width.

\section{Theoretical background}

\subsection{The isoperimetric problem in calculus of variations}

The isoperimetric problem refers to the one to determine the extreme value of the general functional $v$ :

$$
v=\int_{x_{0}}^{x_{1}} F\left(x, y_{1}, y_{2}, \cdots, y_{n}, y_{1}^{\prime}, y_{2}^{\prime}, \cdots, y_{n}^{\prime}\right) d x,
$$

which satisfies the isoperimetric conditions, i.e., the general functions:

$$
\int_{x_{0}}^{x_{1}} \varphi_{i}\left(x, y_{1}, y_{2}, \cdots, y_{n}, y_{1}^{\prime}, y_{2}^{\prime}, \cdots, y_{n}^{\prime}\right) d x=l_{i}, \quad(i=1, \cdots, m),
$$

where $l_{i}$ are all constants. $y_{1}, y_{2}, \cdots, y_{n}$ are functions of $x$ and $y_{1}^{\prime}, y_{2}^{\prime}, \cdots, y_{n}^{\prime}$ are first derivatives of $x$. For illustration and for the purpose of this paper, the cases with the second derivatives or even higher order derivatives are not shown, but they could be added in without affecting this discussion. The ordinal number $m$ can be larger, equal or smaller than the ordinal number $n$. Normally, it is difficult to directly solve these systems of equations as described in Eq. (2.2) since the general functions can be in a very complicated form. By using the Lagrange multiplier method, this type of problem can be transferred to an unconditional extremum problem. In order to ensure this proposition, there should be constant factors $\lambda_{i}(i=1, \cdots, m)$ that make the functions $y_{1}, y_{2}, \cdots, y_{n}$ become the unconditional extremum solutions to the general functional $v^{*}$ :

$$
v^{*}=\int_{x_{0}}^{x_{1}}\left(F+\sum_{i=1}^{m \mid} \lambda_{i} \varphi_{i}\right) d x=\int_{x_{0}}^{x_{1}} F^{*} d x
$$

The function $y_{j}(j=1,2, \cdots, n)$ can be determined by the Euler-Lagrange equation:

$$
\frac{\partial F^{*}}{\partial y_{j}}-\frac{d}{d x} \frac{\partial F^{*}}{\partial y_{j}^{\prime}}=0
$$

or

$$
\frac{\partial F}{\partial y_{j}}+\sum_{i=1}^{m} \lambda_{i} \frac{\partial \varphi_{i}}{\partial y_{j}}-\frac{d}{d x}\left(\frac{\partial F}{\partial y_{j}^{\prime}}+\sum_{i=1}^{m} \lambda_{i} \frac{\partial \varphi_{i}}{\partial y_{j}^{\prime}}\right)=0, \quad(j=1,2, \cdots, n),
$$


The arbitrary constants in general solutions and the values of constant $\lambda_{i}, \lambda_{2}, \cdots, \lambda_{m}$ can be determined by Eq. (2.5) together with the boundary conditions:

$$
y_{j}\left(x_{0}\right)=y_{j_{0}}, \quad y_{j}\left(x_{1}\right)=y_{j_{1}} \quad(j=1,2, \cdots, n),
$$

and the isoperimetric conditions equation $(2.2)[1,10]$.

\subsection{Formulation of analytical equations}

The Euler-Bernoulli beam theory is based on the three assumptions that the cross-section is fully rigid for shear deformation on its plane, the cross-section remains plane, and the cross-section remains perpendicular to the longitudinal axis after bending, as depicted in Fig. 2. This assumption results in zero transverse shear strain. The Euler-Bernoulli beam (or simply Euler beam) is also called engineering beam or thin beam in engineering practices. If the plane cross sections still remain plane but not necessarily normal to the longitudinal axis after bending, the shear strain will not be zero essentially. A specific angle $\varphi_{z}$ as an additional variable will be needed, which is different from the Euler beam (with $\varphi_{z}=0$ always). This specific beam is called Timoshenko beam, as shown in Fig. 2. The Timoshenko beam is also called shear deformation beam or thick beam in engineering practices.

It should be mentioned that the analytical equations can be obtained to determine the free end deflection of the cantilever beam by solving the governing differential equations with the boundary conditions (called strong form in the Finite Element context). The double integral form together with additional terms consisting of initially unknown constants makes the problem complicated for sections with varying second moment of area especially when the target is to obtain an analytical solution. However, the energy theorem is more convenient for these types of problems since only scalars are treated and different loading conditions are dealt with in a unified manner.

To formulate the analytical solutions to the deflection of the free end loadings of a tapered cantilever beam, the energy theorem is adopted with the assumption of small displacements in this paper. Specifically, Castigliano's second theorem is very useful for this purpose and it is valid only for linear elastic materials, which is the assumed case for this

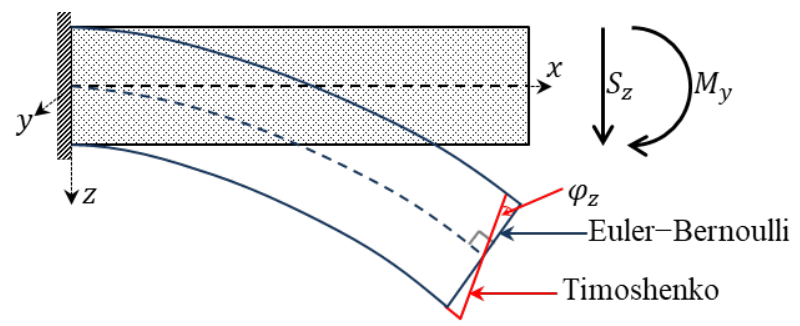

Figure 2: Schematic view of deflection by Euler beam and Timoshenko beam. 
paper. This theorem states that, for a linear elastic structure, the first partial derivative of the strain energy $U$ with respect to any one of generalized forces $Q_{i}$ (Force/Moment) is equal to the corresponding displacement $q_{i}$, as expressed mathematically in Eq. (2.7)

$$
\frac{\partial U}{\partial Q_{i}}=q_{i}
$$

where the strain energy $U$ over the volume $V$ can be expressed by Eq. (2.8) with Einstein's summation convention used

$$
U=\int_{V}^{i}\left(\int_{0}^{\epsilon_{i j}} \sigma_{i j} d \epsilon_{i j}\right) d V
$$

where $\sigma_{i j}$ and $\varepsilon_{i j}$ are the Cauchy stress tensor components and small strain components respectively, both with respect to a Cartesian coordinate system. Castigliano's second theorem can be derived from the well-established energy principle called principle of minimum complementary energy, which is valid for both linear and nonlinear elastic materials and which takes a similar form to Eq. (2.7) by replacing the strain energy $U$ by the complementary energy $U^{*}$, expressed by Eq. (2.9)

$$
U^{*}=\int_{V}^{H}\left(\int_{0}^{\sigma_{i j}} \epsilon_{i j} d \sigma_{i j}\right) d V .
$$

The total strain energy of a linear elastic material due to bending effect and shear effect can be derived as in Eqs. (2.10a) and (2.10b), respectively

$$
\begin{aligned}
& U_{m}=\int_{0}^{L} \frac{M^{2}}{2 E I} d x, \\
& U_{s}=\int_{0}^{L} \frac{k S^{2}}{2 G A} d x
\end{aligned}
$$

where $M$ is the bending moment, $E$ is the elastic modulus, $I$ is the second moment of area, $S$ is the shear force, $G$ is the shear modulus, $A$ is the cross-sectional area and $k$ is the shape factor.

For linear elastic materials, total stored strain energy due to bending is given by Eq. (2.10a). According to the fundamental concept of Castigliano's second theorem, the partial derivative of strain energy with respect to the force applied at the free end of the beam will give the deflection at the free end. For a concentrated load $P$ applied at the free end, Eq. (2.10a) is simplified to Eq. (2.11), which gives the free end deflection $\delta_{e}$

$$
\delta_{s}=\frac{\partial U_{s}}{\partial P}=\frac{6}{5 G} \int_{0}^{L} \frac{S}{A} \frac{\partial S}{\partial P} d x=\frac{6}{5 G} \int_{0}^{L} \frac{P}{A} \frac{\partial P}{\partial P} d x=\frac{6 P}{5 G} \int_{0}^{L} \frac{1}{A} d x .
$$

On the other hand, total stored strain energy due to shear is given by Eq. (2.10b). The shape correction factor, $k$, is taken as 1.2 which is the exact value for a rectangular or 
square shape cross section and independent of other properties of the material or dimensions. In a similar approach to the bending and the fundamental concept of Castigliano's theorem, the partial derivative of strain energy with respect to the force applied at the free end of the beam will give the free end deflection due to the shear. For a concentrated load of $P$ applied at the free end, Eq. (2.10b) is simplified to Eq. (2.12) which gives the free end deflection by shear $\delta_{s}$

$$
\delta_{s}=\frac{\partial U_{s}}{\partial P}=\frac{6}{5 G} \int_{0}^{L} \frac{S}{A} \frac{\partial S}{\partial P} d x=\frac{6}{5 G} \int_{0}^{L} \frac{P}{A} \frac{\partial P}{\partial P} d x=\frac{6 P}{5 G} \int_{0}^{L} \frac{1}{A} d x .
$$

This concept can be expanded to determine free end deflections under any given loading condition. More details of energy theorems can be found in Tauchert [15] and Washizu [17] among many others.

\section{Variational derivation}

\subsection{Problem statement}

For a cantilever beam with a definite tip point load and end displacement, how to select the tapering curve and the material that can minimize its mass is of practical importance, assuming that the cross section remains rectangular. In this section, the width variational curve of the cantilever tapered beam that can minimize the mass is derived based on the theory mentioned in Section 2. Although the width is changing, the height remains constant and the cross section of the beam remains rectangular, as shown in Fig. 1 and Fig. 3.

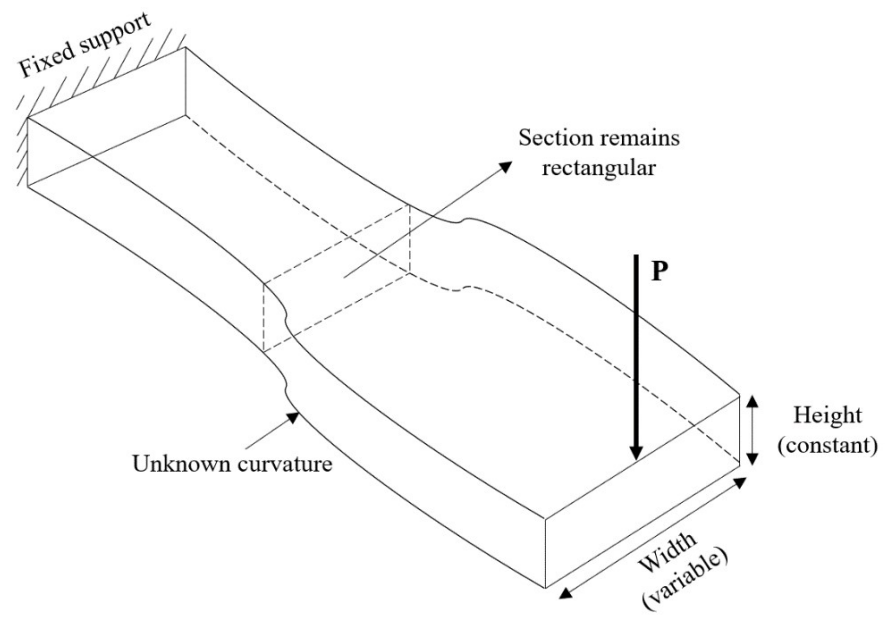

Figure 3: Schematic diagram of a cantilever beam only tapered for the width. 
A concentrated load of $P$ is applied at the tip end. The tip deflection is set as a fixed value (the value is usually specified by design requirement in terms of the Serviceability Limit State). Two different beam types are taken into account, i.e., the Euler-Bernoulli beam without shear deformation and the Timoshenko beam with shear deformation. Furthermore, the final derived curve is the combination of two boundaries of beam width. The final shape of beam depends on how the variation curve is allocated to the two boundaries of beam width. The minimum mass for each case is calculated with recommendations of material selections. The material properties of the beam are assumed to be homogeneous and isotropic.

\subsection{Deviation for Euler-Bernoulli beam type}

The mass of the beam $\mathrm{m}$ can be calculated by Eq. (3.1)

$$
m=\rho \int_{0}^{L} A(x) d x .
$$

Since the rectangular cross-section has constant height $h$ and variable width $b$, the section area and second moment of area is given by Eqs. (3.2a) and (3.2b)

$$
\begin{aligned}
& A(x)=b(x) h, \\
& I(x)=\frac{1}{12} b(x) h^{3} .
\end{aligned}
$$

Substitute them into Eqs. (2.11) and (3.1) and simplify:

$$
\begin{aligned}
& \int_{0}^{L} \frac{x^{2}}{b(x)} d x=\frac{E h^{3} \delta_{e}}{12 P}, \\
& m=\rho h \int_{0}^{L} b(x) d x .
\end{aligned}
$$

Then, the problem becomes an isoperimetric problem with the target that the mass (Eq. (3.3b)) could take the minimum value under the isoperimetric condition equation (3.3a), where $b(x)$ is the extremal function to be solved. Introduce an auxiliary functional:

$$
F^{*}=b+\lambda \frac{x^{2}}{b}, \text { i.e., } m^{*}=\rho h \int_{0}^{L} F^{*} d x .
$$

Use the Euler-Lagrange equation:

$$
\frac{\partial F^{*}}{\partial b}=1-\lambda \frac{x^{2}}{b^{2}}=0, \quad b=\sqrt{\lambda} x .
$$

Substitute this equation into the isoperimetric condition equation (3.3a) and simplify:

$$
\sqrt{\lambda}=\frac{6 l^{2} P}{E h^{3} \delta_{e}}, \text { i.e., } b(x)=\frac{6 l^{2} P}{E h^{3} \delta_{e}} x .
$$


Meanwhile, the minimum mass can be calculated as:

$$
m=\rho h \int_{0}^{L} b(x) d x=\rho h \int_{0}^{L} \frac{6 l^{2} P}{E h^{3} \delta_{e}} x d x=\frac{3 l^{4} P}{h^{2} \delta_{e}} \cdot \frac{\rho}{E} .
$$

\subsection{Deviation for Timoshenko beam type}

The total deflection for the Timoshenko beam is given by Eq. (3.8)

$$
\delta_{t o t}=\delta_{e}+\delta_{s}=\frac{P}{E} \int_{0}^{L} \frac{x^{2}}{I} d x+\frac{6 P}{5 G} \int_{0}^{L} \frac{1}{A} d x
$$

The mass of the beam $\mathrm{m}$ can be calculated similar to Eq. (3.3b). The section area and second moment of area can also refer to Eqs. (3.2a) and (3.2b), respectively. Substitute them into Eq. (3.8) and simplify:

$$
\int_{0}^{L}\left(\alpha \cdot \frac{x^{2}}{b(x)}+\beta \cdot \frac{1}{b(x)}\right) d x=\delta_{\text {tot }}, \quad \text { where } \quad \alpha=\frac{12 P}{E h^{3}} \beta=\frac{6 P}{5 G h} .
$$

Then, the problem becomes an isoperimetric problem with the target that the mass (Eq. (3.3b)) could take the minimum value under the isoperimetric condition equation (3.9), where $b(x)$ is the extremal function to be solved. Introduce an auxiliary functional:

$$
F^{*}=b+\lambda\left(\frac{\alpha x^{2}}{b}+\frac{\beta}{b}\right), \quad m^{*}=\rho h \int_{0}^{L} F^{*} d x .
$$

Use the Euler-Lagrange equation:

$$
\frac{\partial F^{*}}{\partial b}=1-\lambda \frac{\left(\alpha x^{2}+\beta\right)}{b^{2}}=0, \quad b=\sqrt{\lambda} \sqrt{\alpha x^{2}+\beta} .
$$

Substitute this equation into the isoperimetric condition equation (3.9) and simplify:

$$
\begin{aligned}
& \sqrt{\lambda}=\frac{1}{2 \delta_{t o t}}\left(L \sqrt{\alpha L^{2}+\beta}+\frac{\beta}{\sqrt{\alpha}} \ln \left(\sqrt{\frac{\alpha}{\beta} L^{2}+1}+\sqrt{\frac{\alpha}{\beta}} L\right)\right) \\
& \text { i.e., } b(x)=\frac{1}{2 \delta_{t o t}}\left(L \sqrt{\alpha L^{2}+\beta}+\frac{\beta}{\sqrt{\alpha}} \ln \left(\sqrt{\frac{\alpha}{\beta} L^{2}+1}+\sqrt{\frac{\alpha}{\beta}} L\right)\right) \sqrt{\alpha x^{2}+\beta} .
\end{aligned}
$$

Meanwhile, the minimum mass can be calculated as:

$$
\begin{gathered}
m=\rho h \delta_{\text {tot }}\left(L \sqrt{\alpha L^{2}+\beta}+\frac{\beta}{\sqrt{\alpha}} \ln \left(\sqrt{\frac{\alpha}{\beta} L^{2}+1}+\sqrt{\frac{\alpha}{\beta}} L\right)\right)^{2}, \\
\text { where } \alpha=\frac{12 P}{E h^{3}} \beta=\frac{6 P}{5 G h} .
\end{gathered}
$$




\section{Discussion}

\subsection{Optimization in terms of materials}

According to Eq. (3.6), the optimized width variation curve for the Euler-Bernoulli beam is shown to be a linear function of $x$. The final result of the mass in Eq. (3.7) indicates that the ratio $\rho / E$ should be as small as possible when selecting the materials to minimize the mass, if the tip deflection is kept at a given value. For the Timoshenko beam, the optimized width variation curve is shown to be a nonlinear function of $x$ in Eq. (3.12). However, the coefficients in the equation are relatively complicated and not easy to obtain. It is therefore also difficult to determine how to select the materials to minimize the mass in Eq. (3.13) directly. To circumvent this, a comparative study is conducted between the Euler-Bernoulli beam and the Timoshenko beam by using the aluminum alloy as an example. The beam parameters are assumed as shown in Table 1. The height against length ratio is assumed to be larger than $1 / 4$ to model the Timoshenko beam. Three alternative values are set for the Poisson' ratio in order to investigate how the degree of shear deformation affects the optimization of tapered cantilever beams.

Fig. 4 is created based on Eqs. (3.6) and (3.12) by use of the beam parameters in Table 2. The curves in Fig. 4 show the optimized beam width as functions of the longitudinal location given the specified conditions. The projected area of these curves to abscissa multiplied by the constant height reflects the volume of the beam, and further reflects the minimum mass and material consumption for each case. Comparing the Euler-Bernoulli beam to the Timoshenko beams, it can be found that with the increase of Poisson's ratio, the required minimum mass increases, noting also that Euler-Bernoulli beam can be treated as a special degenerated case of Timoshenko beam with a Poisson's ratio $v=-0.999 \ldots$ (with the shear modulus approaching infinity). In this sense, the optimized mass for the case of Euler-Bernoulli beam is the lower boundary limit which the Timoshenko case cannot go beyond. Meanwhile, for each Timoshenko beam case, the initial beam width starts from non-zero due to the shear effect, against the Euler-Bernoulli beam case (with starting beam width of zero), which can be seen from Eq. (3.12), in which the starting beam width $(x=0)$ and the non-zero value of $\beta$ lead to a non-zero value of

Table 1: Assumed beam parameters.

\begin{tabular}{||c|c|c||}
\hline Parameter & Value & Unit \\
\hline Beam length & 1 & $\mathrm{~m}$ \\
\hline Beam height & 0.3 & $\mathrm{~m}$ \\
\hline Elastic modulus & 66 & $\mathrm{GPa}$ \\
\hline Poisson's ratio & $0.0 / 0.25 / 0.5$ & - \\
\hline End deflection & 0.02 & $\mathrm{~m}$ \\
\hline Density & 2700 & $\mathrm{~kg} / \mathrm{m}^{3}$ \\
\hline End Point load & 1000 & $\mathrm{kN}$ \\
\hline
\end{tabular}




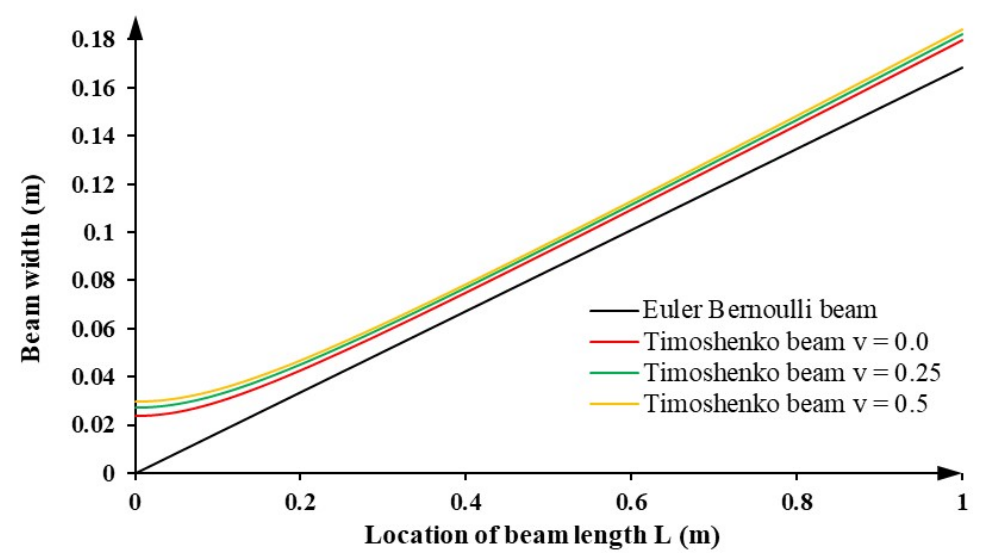

Figure 4: Width variation curves for the Euler-Bernoulli beam and Timoshenko beams with different Poisson's ratios (Aluminum alloy).

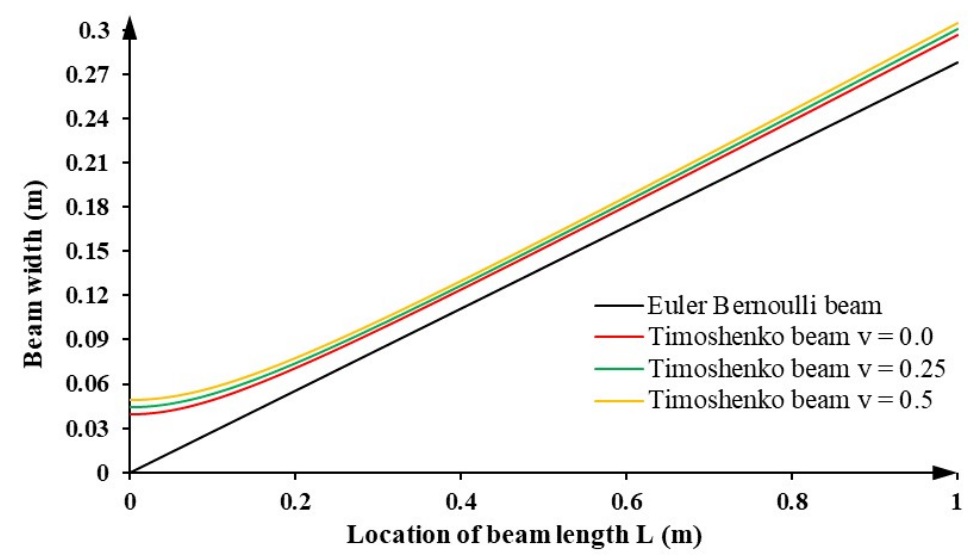

Figure 5: Width variation curves for the Euler-Bernoulli beam and Timoshenko beams with different Poisson's ratios (Magnesium alloy).

$b(0)$ for the Timoshenko beam case. However, the latter variation tendency of them is somewhat similar to that of the Euler-Bernoulli beam. This phenomenon can also be found for other materials, for instance, the magnesium alloy (Elastic modulus 40GPa \& Density $1800 \mathrm{~kg} / \mathrm{m}^{3}$ ), as shown in Fig. 5 .

Furthermore, the minimum masses for each case are calculated in Table 2. It is indicated that the projection for each beam made of magnesium alloy is apparently larger than that made of aluminum alloy. Combining the beams using different materials in Fig. 4 and Fig. 5, it can be seen that the tendency of Euler-Bernoulli beam and the Timoshenko beam is similar except for the initial difference and their similarity is independent of the material used. This indicates that the conclusion "to minimize the mass, the ratio 
$\rho / E$ should be as small as possible" is also applicable for the Timoshenko beam for the assumed scenario.

\subsection{Optimization in terms of height-to-length ratio}

To investigate the effect of height-to-length ratio on the optimization of the tapered cantilever beam, the ratio (square) is defined as $\zeta$. Specific power should be added for the ratio in order to make use of Eq. (3.7)

$$
\zeta=h^{2} / L^{4}
$$

Three different values of the ratios are assumed, i.e., 0.1, 0.2 and 0.3. To control the variable, the lengths of all the beams are all set to be $1 \mathrm{~m}$ and the Poisson's ratio is a constant 0.2. The other parameters are chosen from Table 2 except the height (variable). The optimized curves for Euler-Bernoulli beam and Timoshenko beam with different heightto-length ratios are shown in Fig. 6. It is indicated from Fig. 6 that with the increase of the height-to-length ratio, the slopes of the curves decrease for both Euler-Bernoulli beam and Timoshenko beam. The decreasing rate gradually reduces. For the same ratio but with different beam types, the variation rule is very similar to that discussed in section 4.1 for optimization in terms of materials. Additionally, the minimum masses for each case are calculated in Table 3. The required minimum mass is larger with the smaller value of $\zeta$.

Finally, it should be mentioned that the width variation curves shown above only govern the overall variation. Since one beam has two boundaries for its width, the derived curves are the combination of the two boundaries. In other words, the final shape of beam depends on how the variation curve is allocated to the two boundaries of beam width too. The scenarios set in Figs. 4-6 equivalently assumes one width boundary keeping horizontal and another one varies along the optimized curve.

Table 2: The minimum mass of tapered beams with different beam theory and materials.

\begin{tabular}{||c|c|c|c|c||}
\hline \multirow{2}{*}{ Material } & \multicolumn{4}{|c||}{ Minimum mass (kg) } \\
\cline { 2 - 5 } & \multirow{2}{*}{ Euler-Bernoulli beam } & \multicolumn{3}{|c||}{ Timoshenko beam with Poisson's ratio } \\
\cline { 3 - 5 } & & $v=0$ & $v=0.25$ & $v=0.5$ \\
\hline Aluminum alloy & 68.18 & 76.27 & 78.00 & 79.68 \\
\hline Magnesium alloy & 75.00 & 83.90 & 85.80 & 87.65 \\
\hline
\end{tabular}

Table 3: The minimum mass of tapered beams with different theory and ratios.

\begin{tabular}{||c|c|c|c|c|c|c||}
\hline Beam theory & \multicolumn{2}{|c|}{ Euler-Bernoulli beam } & \multicolumn{3}{c||}{ Timoshenko beam } \\
\hline Ratio $(\zeta)$ & 0.1 & 0.2 & 0.3 & 0.1 & 0.2 & 0.3 \\
\hline Minimum mass $(\mathrm{kg})$ & 61.36 & 30.68 & 20.45 & 70.71 & 39.2 & 28.54 \\
\hline
\end{tabular}




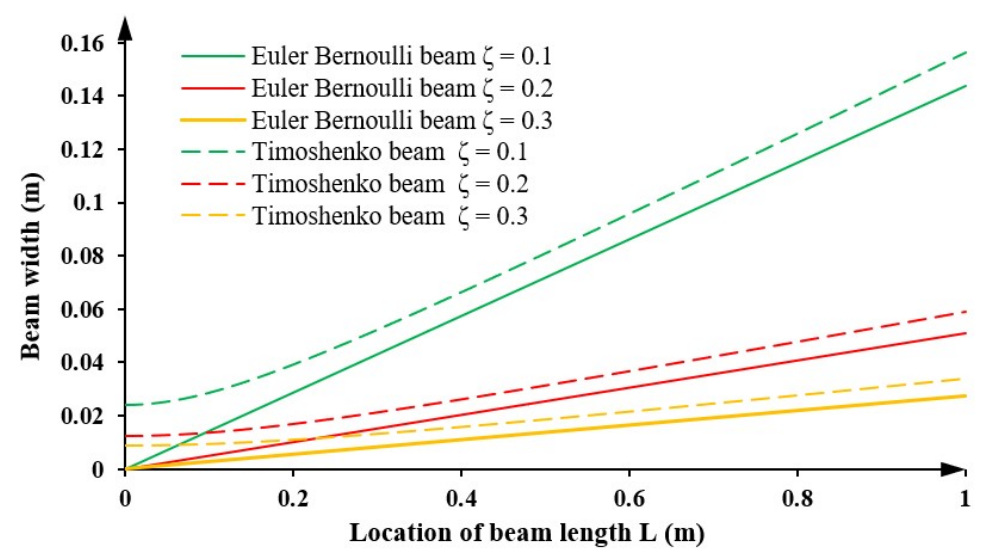

Figure 6: Width variation curves for the Euler-Bernoulli beam and Timoshenko beams with different height-tolength ratios (aluminum alloy).

\subsection{Extension of the study}

In the previous sections, the optimization scheme only solves the situation where a cantilever beam with variable width is subjected to a tip point load. Actually, the procedure of applying the variational method to beam optimization is applicable for many other cases. For example, the variable can be extended to width, height and even the crosssection shape and the load types may include the tip point load (TPL) and the uniformly distributed load (UDL, force/length), or even an arbitrary load condition. Some of these cases as well as the solutions are shown in Table 4.

It can be seen from Table 4 that for the Euler-Bernoulli beam type, the optimized curves are all power functions of length $x$ for all cases. The analytical solutions for the Timoshenko beam type are complicated when determining the constant $\lambda$ set up in the Euler-Lagrange equation and the exact expression of $\lambda$ is not provided here. One of the derivation processes for Case 6a is presented in Appendix.

\section{Conclusions}

In summary, this study focuses on using the variational method to determine the minimum mass and corresponding optimized shape of a cantilever tapered beam under a tip load and with a fixed end displacement. Both the Euler-Bernoulli beam and Timoshenko beam types are considered and compared in the investigation. Based on the derivation and discussion, the following conclusions can be made:

- The optimized curve of beam width for the Euler-Bernoulli beam is a linear function of longitudinal location (with the starting width of zero), but nonlinear for the Timoshenko beam. The curve of the Timoshenko beam starts from non-zero at the 
Table 4: Summary of the solutions.

\begin{tabular}{|c|c|c|c|c|c|}
\hline No. & Variable & Constant & Beam type & Load type & Optimized Curve \\
\hline Case $1 \mathrm{a}$ & Width & Height & $\begin{array}{c}\text { Euler- } \\
\text { Bernoulli }\end{array}$ & TPL & $b(x)=\frac{6 l^{2} P}{E h^{3} \delta_{e}} x$ \\
\hline Case $1 b$ & Width & Height & Timoshenko & TPL & $\begin{array}{l}b(x)=\sqrt{\lambda} \sqrt{\alpha x^{2}+\beta}, \text { where } \\
\alpha=\frac{12 P}{E h^{3}}, \beta=\frac{6 P}{5 G h}, \lambda \text { related to } \alpha, \beta, l, \delta_{\text {tot }}\end{array}$ \\
\hline Case $2 \mathrm{a}$ & Width & Height & $\begin{array}{l}\text { Euler- } \\
\text { Bernoulli }\end{array}$ & UDL & $b(x)=\frac{12 l^{5 / 2} q}{5 E h^{3} \delta_{e}} x^{3 / 2}$ \\
\hline Case $2 b$ & Width & Height & Timoshenko & UDL & $\begin{array}{l}b(x)=\sqrt{\lambda} \sqrt{\alpha x^{3}+\beta x}, \text { where } \\
\alpha=\frac{6 q}{E h^{3}}, \beta=\frac{6 q}{5 G h}, \lambda \text { related to } \alpha, \beta, l, \delta_{\text {tot }}\end{array}$ \\
\hline Case 3a & Height & Width & $\begin{array}{c}\text { Euler- } \\
\text { Bernoulli }\end{array}$ & TPL & $h(x)=\frac{2 l^{1 / 2} P^{1 / 3}}{E^{1 / 3} b^{1 / 3} \delta_{e}^{1 / 3}} x^{1 / 2}$ \\
\hline Case $3 b$ & Height & Width & Timoshenko & TPL & $\begin{array}{l}h(x)=\sqrt{\frac{\lambda \beta}{2}+\frac{\lambda}{2} \sqrt{\beta^{2}+\frac{12 \alpha x^{2}}{\lambda}}}, \text { where } \\
\alpha=\frac{12 P}{E b}, \beta=\frac{6 P}{5 G b}, \lambda \text { related to } \alpha, \beta, l, \delta_{\text {tot }}\end{array}$ \\
\hline Case $4 a$ & Height & Width & $\begin{array}{l}\text { Euler- } \\
\text { Bernoulli }\end{array}$ & UDL & $h(x)=\frac{24^{1 / 3} l^{7 / 12} q^{1 / 3}}{7^{1 / 3} E^{1 / 3} b^{1 / 3} \delta_{e}^{1 / 3}} x^{3 / 4}$ \\
\hline Case $4 b$ & Height & Width & Timoshenko & UDL & $\begin{array}{l}h(x)=\sqrt{\frac{\lambda \beta x}{2}+\frac{\lambda x}{2} \sqrt{\beta^{2}+\frac{12 \alpha x}{\lambda}}}, \text { where } \\
\alpha=\frac{6 q}{E b}, \beta=\frac{6 q}{5 G b}, \lambda \text { related to } \alpha, \beta, l, \delta_{\text {tot }}\end{array}$ \\
\hline Case $5 a$ & $\begin{array}{l}\text { Section } \\
\text { size }\end{array}$ & $\begin{array}{c}h / b= \\
\text { constant } k\end{array}$ & $\begin{array}{l}\text { Euler- } \\
\text { Bernoulli }\end{array}$ & TPL & $A(x)=\frac{6 l^{5 / 6} P^{1 / 2}}{5^{1 / 2} E^{1 / 2} k^{1 / 2} \delta_{e}^{1 / 2}} x^{2 / 3}$ \\
\hline Case $6 a$ & $\begin{array}{l}\text { Section } \\
\text { size }\end{array}$ & $\begin{array}{c}h / b= \\
\text { constant } k\end{array}$ & $\begin{array}{l}\text { Euler- } \\
\text { Bernoulli }\end{array}$ & UDL & $A(x)=\frac{3^{1 / 2} l q^{1 / 2}}{E^{1 / 2} k^{1 / 2} \delta_{e}^{1 / 2}} x$ \\
\hline
\end{tabular}

(Note: $A(x)$ is the area of cross-section. $P$ and $q$ are the magnitude of TPL and UDL, respectively. Section remains rectangular.)

tip end, but its tendency gradually approaches the one of the Euler-Bernoulli beam. The starting non-zero value for the Timoshenko beam increases with the ratio $\zeta$ decreasing.

- With the increase in the Poisson's ratio, the required minimum mass of the beam will increase however the material changes. The optimized mass for the case of Euler-Bernoulli beam is the lower boundary limit which the Timoshenko case cannot go beyond.

- The ratio $\rho / E$ of the material should be as small as possible, while the ratio $\zeta$ of the beam should be as large as possible in order to minimize the mass for EulerBernoulli beam. This conclusion is also applicable for the Timoshenko beam.

- The optimized curves for Euler-Bernoulli beam types are all found to be power functions of length for TPL cases and for UDL cases. 


\section{Appendix}

For Case 6a in Table 4, the derivation is provided below.

For a cantilever beam with a uniformly distributed load, a definite end displacement and similar cross-section $(h / b=$ constant), how to select the tapering curve of crosssectional area and the material that can minimize its mass is of interest, assuming that the cross section remains rectangular and the Euler-Bernoulli beam type is considered.

For a uniformly distributed load $(q)$ applied, the free end displacement due to bending derived from the total strain energy is given by Eq. (A.1)

$$
\delta_{e}=\frac{q}{2 E} \int_{0}^{L} \frac{x^{3}}{I(x)} d x
$$

The mass of the beam $m$ can be calculated by Eq. (A.2)

$$
m=\rho \int_{0}^{L} A(x) d x
$$

Since the cantilever tapered beam has similar cross-section, i.e., $h / b$ is constant $k$, the second moment of area can be rewritten in terms of cross-sectional area $A(x)$ in Eq. (A.3)

$$
I(x)=\frac{k}{12} A^{2}(x)
$$

Substitute it into Eq. (A.1) and simplify:

$$
\int_{0}^{L} \frac{x^{3}}{A^{2}(x)} d x=\frac{k E \delta_{e}}{6 q}
$$

Then, the problem becomes an isoperimetric problem with the target that the mass (Eq. (A.2)) could take the minimum value under the isoperimetric condition equation (A.4), where $A(x)$ is the extremal function to be solved. Introduce an auxiliary functional:

$$
F^{*}=A+\lambda \frac{x^{3}}{A^{2}}, \quad \text { i.e., } m^{*}=\rho \int_{0}^{L} F^{*} d x .
$$

Use the Euler-Lagrange equation:

$$
\frac{\partial F^{*}}{\partial A}=1-2 \lambda \frac{x^{3}}{A^{3}}=0, \quad A=(2 \lambda)^{1 / 3} x .
$$

Substitute this equation into the isoperimetric condition equation (A.4) and simplify:

$$
(2 \lambda)^{1 / 3}=\frac{3^{1 / 2} q^{1 / 2} l}{k^{1 / 2} E^{1 / 2} \delta_{e}^{1 / 2}}, \quad \text { i.e., } A(x)=\frac{3^{1 / 2} q^{1 / 2} l}{k^{1 / 2} E^{1 / 2} \delta_{e}^{1 / 2}} x .
$$


Meanwhile, the minimum mass can be calculated as:

$$
m=\rho \int_{0}^{L} A(x) d x=\rho \int_{0}^{L} \frac{3^{1 / 2} q^{1 / 2} l}{k^{1 / 2} E^{1 / 2} \delta_{e}^{1 / 2}} x d x=\frac{3^{1 / 2} q^{1 / 2} l^{3}}{2 k^{1 / 2} \delta_{e}^{1 / 2}} \cdot \frac{\rho}{E^{1 / 2}} .
$$

It can be seen from Eq. (A.8) that in this particular case, the ratio $\rho / E^{(1 / 2)}$ should be as small as possible when selecting the materials to minimize the mass.

\section{Acknowledgements}

The funding supports from Xi'an Jiaotong-Liverpool University (RDF 14-02-44, RDF 1501-38, RDF 18-01-23 and PGRS1906002) and the Key Program Special Fund at XJTLU (Grant No. KSF-E-19) are gratefully acknowledged.

\section{References}

[1] W. G. CHEN, Variational Principle of Mechanics, Shanghai: Tongii University Press, (1989).

[2] J. M. DieTL, AND E. GARCiA, Beam shape optimization for power harvesting, J. Intell. Mater Syst. Struct., 21(6) (2010), pp. 633-646.

[3] G. GonG, AND J. XIA, A revisit to higher variations of a functional, J. Adv. Res. Math. Stat., 6(2) (2019), pp. 1-15.

[4] R. O. GROSSI, AND B. D. ARENAS, A variational approach to the vibration of tapered beams with elastically restrained ends, J. Sound Vib., 195(3) (1996), pp. 507-511.

[5] P. Hajheidari, I. Stiharu, AND R. Bhat, Performance of tapered cantilever piezoelectric energy harvester based on Euler-Bernoulli and Timoshenko Beam theories, J. Intell. Mater Syst. Struct., 31(4) (2019), pp. 487-502.

[6] L. H. HARPER, Global Methods for Combinatorial Isoperimetric Problems, Cambridge: Cambridge University Press, (2004).

[7] M. H. IMAM, Three-dimensional shape optimization, Int. J. Numer. Methods Eng., 18 (1982), pp. 661-673.

[8] J. KatsiKadelis, AND G. Tsiatas, Buckling load optimization of beams, Arch. Appl. Mech., 74(11-12) (2005), pp. 790-799.

[9] Y. Y. KIM, AND T. S. KIM, Topology optimization of beam cross sections, Int. J. Solids Struct., 37(3) (2000), pp. 477-493.

[10] L. KomZsIK, Applied Calculus of Variations for Engineers, Boca Raton: CRC Press, (2009).

[11] G. Q. LI, AND J. J. LI, A tapered Timoshenko-Euler beam element for analysis of steel portal frames, J. Constr. Steel Res., 58 (2002), pp. 1531-1544.

[12] M. OHSAKI, H. TAGAWA, AND P. PAN, Shape optimization of reduced beam section under cyclic loads, J. Constr. Steel Res., 65(7) (2009), pp. 1511-1519.

[13] M. A. Rosa, AND N. M. Auciello, Free vibrations of tapered beams with flexible ends, Comput. Struct., 60(2) (1996), pp. 197-202.

[14] P. K. ROY, AND N. GANESAN, Some studies on the response of a tapered beam, Comput. Struct., 45(I) (1992), pp. 18-195.

[15] T. TAUCHERT, Energy Principles in Structural Mechanics, New York: McGraw-Hill, Inc, (1974). 
[16] P. VINOT, S. COGAN, AND J. PIRANDA, Shape optimization of thin-walled beam-like structures, Thin-Walled Struct., 39(7) (2001), pp. 611-630.

[17] K. WAshizu, Variational Methods in Elasticity and Plasticity, Oxford: Pergamon Press, (1968).

[18] H. H. Yoo, J. E. Cho, and J. Chung, Modal analysis and shape optimization of rotating cantilever beams, J. Sound Vib., 290(1-2) (2006), pp. 223-241. 